\title{
Occipital Transcranial Magnetic Stimulation Has an Activity-Dependent Suppressive Effect
}

\author{
Francesca Perini, ${ }^{1}$ Luigi Cattaneo, ${ }^{1}$ Marisa Carrasco, ${ }^{2}$ and Jens V. Schwarzbach ${ }^{1,3}$ \\ ${ }^{1}$ Center for Mind/Brain Sciences, University of Trento, 38100 Mattarello (TN), Italy, ${ }^{2}$ Department of Psychology and Center for Neural Science, New York \\ University, New York, New York 10003, and ' ${ }^{3}$ epartment of Cognitive Science and Education, University of Trento, 38068 Rovereto, Italy
}

\begin{abstract}
The effects of transcranial magnetic stimulation (TMS) vary depending on the brain state at the stimulation moment. Four mechanisms have been proposed to underlie these effects: (1) virtual lesion-TMS suppresses neural signals; (2) preferential activation of less active neuronsTMS drives up activity in the stimulated area, but active neurons are saturating; (3) noise generation-TMS adds random neuronal activity, and its effect interacts with stimulus intensity; and (4) noise generation-TMS adds random neuronal activity, and its effect depends on TMS intensity. Here we explore these hypotheses by investigating the effects of TMS on early visual cortex by assessing the contrast response function while varying the adaptation state of the observers. We tested human participants in an orientation discrimination task, in which performance is contingent upon contrast sensitivity. Before each trial, neuronal activation of visual cortex was altered through contrast adaptation to two flickering gratings. In a factorial design, with or without adaptation, a single TMS pulse was delivered simultaneously with targets of varying contrast. Adaptation decreased contrast sensitivity. The effect of TMS on performance was state dependent: TMS decreased contrast sensitivity in the absence of adaptation but increased it after adaptation. None of the proposed mechanisms can account for the results in their entirety, in particular, for the facilitatory effect at intermediate to high contrasts after adaptation. We propose an alternative hypothesis: TMS effects are activity dependent, so that TMS suppresses the most active neurons and thereby changes the balance between excitation and inhibition.
\end{abstract}

\section{Introduction}

Transcranial magnetic stimulation (TMS) is applied on a cortical region of interest to disrupt the ongoing cerebral activity and to test its causal role in a cognitive task, thus inducing a "virtual lesion" (Walsh and Rushworth, 1999; Harris et al., 2008). Behavioral effects of TMS are not fixed, and their direction is determined by the brain state during stimulation (Silvanto and Muggleton, 2008). Thus, psychophysical paradigms-adaptation or priming-can increase TMS functional resolution and improve predictions about its behavioral consequences (Cattaneo and Silvanto, 2008; Silvanto et al., 2008; Cattaneo et al., 2010). TMS adaptation (TMSa) paradigms predict that TMS improves processing of perceptual attributes that are adapted while decreasing performance for nonadapted attributes. The underlying mechanisms of TMS and TMSa are still debated.

Here we manipulated the brain state using contrast adaptation-a decrease in visual contrast sensitivity produced by repeated exposure to high-contrast stimuli (Dao et al., 2006; Pestilli et al., 2007)—to investigate the mechanisms of TMSa. We assessed how TMS affects the behavioral contrast response func-

\footnotetext{
Received Nov. 23, 2011; revised June 3, 2012; accepted June 22, 2012.

Author contributions: F.P., L.C., M.C., and J.V.S. designed research; F.P. and L.C. performed research; F.P. and J.V.S. analyzed data; F.P., L.C., M.C., and J.V.S. wrote the paper.

This research was supported by the Provincia Autonoma di Trento, the Fondazione Cassa di Risparmio di Trento e Rovereto, and NIH Grant R01 EY016200 to M.C.

The authors declare no competing financial interests.

Correspondence should be addressed to Jens V. Schwarzbach, Center for Mind/Brain Sciences, Palazzo Fedrigotti, Corso Bettini 31, 38068 Rovereto (TN), Italy. E-mail: jens.schwarzbach@unitn.it.

DOI:10.1523/JNEUROSCI.5864-11.2012

Copyright $\odot 2012$ the authors $\quad 0270-6474 / 12 / 3212361-05 \$ 15.00 / 0$
}

tions (CRFs), which depict spike rate (McAdams and Maunsell, 1999) or discrimination performance (Pestilli et al., 2009) as a function of stimulus contrast (Fig. 1a). We tested four hypotheses on how TMS modulates perception. (1) Signal suppression (Naka and Rushton, 1966; Amassian et al., 1989) predicts that TMS decreases the sensitivity of the underlying cortex independent of state, leading to a rightward shift of the CRF regardless of adaptation (Fig. 1b). (2) "Preferential activation of less active neurons" (Silvanto and Muggleton, 2008) predicts that as activity increases, TMS activates signal-bearing neurons less than noninformative neurons. Increasing stimulus contrast causes only informative neurons, not noninformative neurons, to increase their firing rate. At high contrast levels, informative neurons are already highly active, so TMS would preferentially excite noninformative neurons. Hence, TMS would decrease the activation difference between informative and noninformative neurons, and this decrement of signal-to-noise ratio (SNR), and thus performance, would be directly proportional to contrast, modulating the asymptote (Fig. 1c). This argument holds if one assumes suppression of the most active neurons. Due to a floor effect, noninformative neurons cannot be further suppressed, and the SNR would decrease with stimulus contrast, decreasing the asymptote. (3) A noise generation hypothesis predicts different effects of TMS as a function of either stimulus intensity (Simonotto et al., 1997; Ruzzoli et al., 2010) or (4) TMS and stimulus intensity (Schwarzkopf et al., 2011). Based on opposite TMS effects depending on the system's state, it has been argued that noise induction in a nonlinear system leads to stochastic resonance (Stocks, 2000; Schwarzkopf et al., 2011). According to hypothesis 3 , stochastic resonance would result in a nonlinear alteration of 
the effective threshold of near-threshold stimuli: noise would boost representations of stimuli just below threshold, improving sensitivity, but it would reduce representations of stimuli just above threshold decreasing sensitivity (Simonotto et al., 1997; Ruzzoli et al., 2010). A parsimonious explanation predicts the same effect for the CRF after adaptation (Fig. 1d). According to hypothesis 4 , with high TMS intensity, sensitivity should decrease regardless of stimulus intensity, but with low TMS intensity, sensitivity would increase, especially for low stimulus values. To explain previous effects of TMS adaptation, Schwarzkopf et al. (2011) claim that the adaptation effect is akin to reducing TMS intensity. Consequently, stimulating with high intensity after adaptation and stimulating with low intensity without adaptation yield the same prediction (Fig. 1e).

\section{Materials and Methods}

Six participants performed a two-alternativesforced-choice (2AFC) orientation discrimination task, following contrast adaptation to two flickering Gabors with (adapted) or without (nonadapted) adaptation (henceforth referred to as "adaptation blocks" or "no-adaptation blocks"). To assess contrast sensitivity, we measured performance in an orientation discrimination task as a function of stimulus contrast (Nachmias, 1967; Carrasco et al., 2000; Cameron et al., 2002; Herrmann et al., 2010).

The experiment consisted of six different sessions. The first session was only behavioral, with a twofold aim: to reach a stable performance and to determine stimulus parameters so that participants' behavior would encompass the whole CRF to enable testing the different models. TMS was applied, and behavior was measured concomitantly using the parameters obtained in the behavioral session.

Participants. Six healthy participants (four females and two males, aged 26-36 years, including two of the authors, F.P. and L.C.) with no history of neurological or psychiatric illness were recruited for the study. Participants were selected given their ability to detect the presence of phosphenes induced by occipital TMS. Four observers were naive to the purpose of the experiment. All participants underwent a screening for TMS contraindications and a short informal debriefing after each session to assess short-term side effects. All participants gave informed consent before participating in the study. The study was approved by the ethics committee of the University of Trento; participants were treated in accordance with the Helsinki declaration.

Apparatus. Stimuli were presented on a 17 inch gamma-corrected LCD monitor (Dell 1908FP-BLK) in a dimly lit room. The background luminance was set to the middle of the monitor range at $59 \mathrm{~cd} / \mathrm{m}^{2}$. The stimuli were generated and presented on a standard PC using Matlab 2007b (Mathworks) and ASF (Schwarzbach, 2011), an add-on to the Psychophysics Toolbox (Brainard, 1997; Pelli, 1997).

The task was a $2 \mathrm{AFC}$ orientation discrimination task on one of two tilted peripheral Gabors (sinusoidal gratings embedded in a Gaussian distribution; Fig. 2), while fixating a central fixation cross $\left(0.5^{\circ} \times 0.5^{\circ}\right.$ visual angle). Trials were divided in adaptation and no-adaptation blocks of 20 trials each. At the beginning of each adaptation block, there were two vertical Gabors displayed $3^{\circ}$ to the left and right and $1.5^{\circ}$ below the fixation cross, flickering at $7.5 \mathrm{~Hz}$ for 70 s. No-adaptation blocks started with a blank gray screen lasting for b) Unspecific suppression

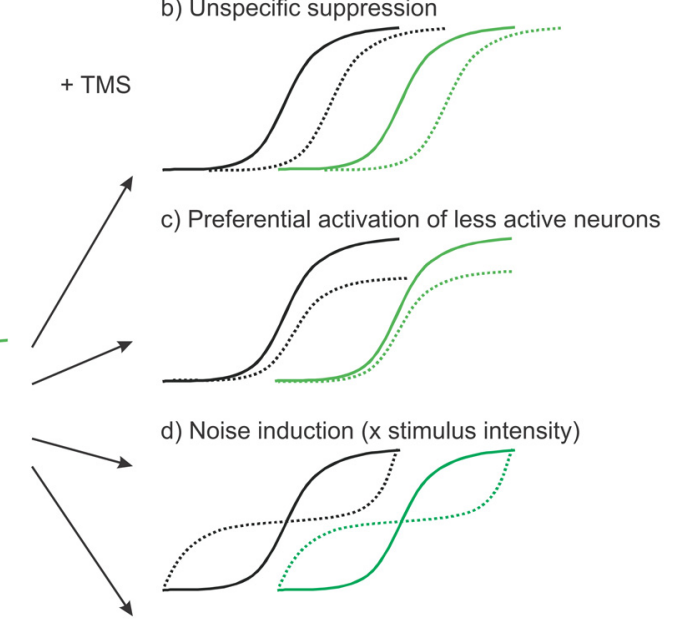

e) Noise induction ( $x$ stimulus- and TMS intensity)

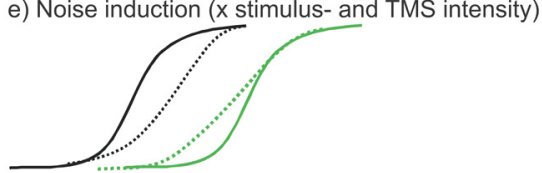

Figure 1. $\boldsymbol{a}-\boldsymbol{e}$, CRF without ( $\boldsymbol{a})$ and with $(\boldsymbol{b}-\boldsymbol{e})$ TMS. $\boldsymbol{a}$, Increasing stimulus contrast leads to increased accuracy of behavioral reports, yielding a CRF (black solid line). Adaptation reduces contrast sensitivity, leading to a rightward shift of the (RF (green solid line). $\boldsymbol{b}-\boldsymbol{e}$

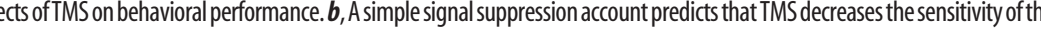

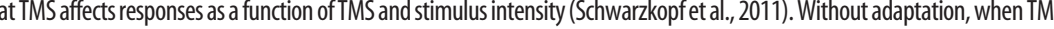
related to contrast. Note that $\boldsymbol{b}-\boldsymbol{d}$ predict qualitatively the same effect of TMS with and without adaptation. In contrast, for $\boldsymbol{e}$ TMS should modulate the shape of the adapted and not adapted CRF in a different way.

$20 \mathrm{~s}$. Each trial started with a $4 \mathrm{~s}$ top-up phase, which consisted of two flickering Gabors in adaptation blocks (and a blank screen with only a fixation cross in no-adaptation blocks). After the presentation of a central white square subtending $1^{\circ} \times 0.4^{\circ}$ visual angle, which served as a fixation point, participants were presented with two static Gabor patches $\left(5\right.$ cycles $/{ }^{\circ}$ subten$\operatorname{ding} 4^{\circ}$ of visual angle in diameter; $\sigma$ of $\left.1.5^{\circ}\right)$. In TMS trials, a single pulse of $120 \%$ of the phosphene threshold was applied at stimulus onset. The test Gabors were displayed at the same locations as the adapting Gabors. To equate participants' performance, the test Gabors were slightly tilted $\left(1.25^{\circ}-\right.$ $2.5^{\circ}$ ), clockwise or counter-clockwise, from vertical. Each Gabor had an independent, randomly chosen tilt. In each trial, both test Gabors had one of six different contrast levels $(2.5,5,10,20,30$, and $80 \%)$. Following presentation of the test Gabors, a response cue (white square) appeared $0.5^{\circ}$ to the left or right of the fixation cross, indicating the side of the target Gabor. Participants responded within a $1 \mathrm{~s}$ allotted time and received acoustic feedback (a high or low pitch for correct or incorrect responses, respectively).

Procedure and design. Participants were trained for $\sim 1 \mathrm{~h}$, and the tilt of orientation was adjusted for each observer and kept constant in the following five TMS sessions. Each of these sessions lasted 90 min: two noTMS runs and two TMS runs. No-TMS and TMS runs were interleaved, and the first run of each session was counterbalanced. Adaptation and no-adaptation blocks were alternated within each run, with half of the runs starting with an adaptation block. In no-TMS runs, the coil was directed to the same location as in TMS runs but moved away from the head by $1-2 \mathrm{~cm}$ such that cortex would not be stimulated, while keeping the somatosensory and auditive experience for the participants constant. Contrast levels of the test Gabors, their respective tilts, and the position of 


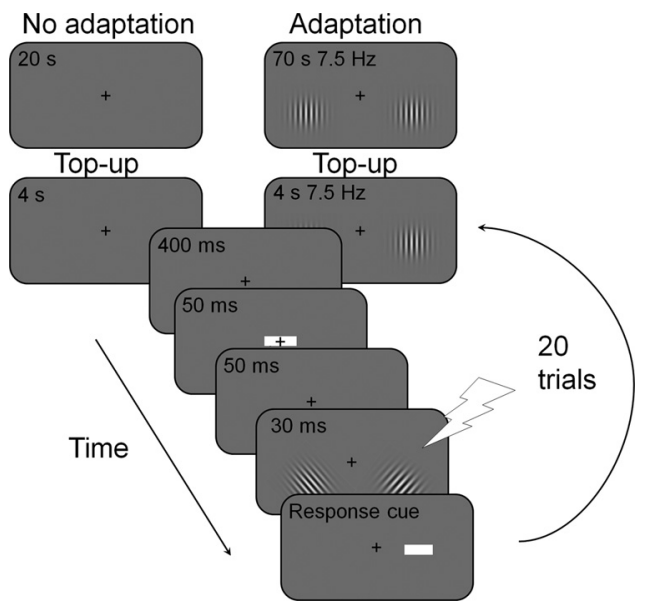

Figure 2. Trial procedure. At the beginning of each block of 20, either adaptation or noadaptation trials, participants were presented with a long adaptation phase. In the noadaptation blocks, participants saw a 20 -s-long blank screen, while fixating the central cross. In the adaptation block, participants saw two vertical Gabors at $100 \%$ contrast flickering for $70 \mathrm{~s}$. After this adaptation phase, there were 20 trials starting with a $4 \mathrm{~s}$ top-up phase. Following the presentation of a $50 \mathrm{~ms}$ central cue and a 50 ms blank screen, two tilted Gabors were presented for 30 ms. In TMS trials, a single pulse of $120 \%$ of the phosphene threshold was applied at stimulus onset. Observers had to discriminate orientation of the target Gabor, whose location was indicated by a subsequent 1 s response cue (task adapted from Pestilli et al., 2007).

the response cue were randomized and counterbalanced throughout the whole experiment. There were 1920 trials per participant, leading to 80 data points per cell and observer.

TMS methods. Before the experiment, a high-resolution T1-weighted scan (magnetization-prepared rapid gradient echo sequence, 176 slices; in-plane resolution $256 \times 224 ; 1 \mathrm{~mm}$ isotropic voxels; generalized autocalibrating partially parallel acquisition with acceleration factor, 2; TR, $2700 \mathrm{~ms}$; TE, $4.18 \mathrm{~ms}$; TI, $1020 \mathrm{~ms}$; flip angle, $7^{\circ}$ ) of the brain of each participant was obtained using a MedSpec 4-T head scanner (BioSpin, Bruker) with an eight-channel array head coil (USA Instruments). We coregistered the position of the TMS coil with the participants' reconstructed head and marked the location of TMS stimulation on the reconstructed pial surface of each individual brain using BrainVoyager Neuronavigator (version 2.1, Brain Innovation BV) combined with a Zebris CMS20S measuring system for real-time motion analysis (Zebris Medical). Average distance between occipital cortex and scalp was 14.5 $\mathrm{mm}$ (range, 11-21 mm).

At the beginning of the first TMS session, we determined the stimulation site by placing the coil over the occipital pole and moving the coil until a single pulse would generate a phosphene at the center of the visual field, where the two Gabors would later be presented. Although the main target of stimulation was V1, surrounding visual areas such as V2 and V3, were also probably stimulated (Thielscher et al., 2010; SalminenVaparanta et al., 2012). The coil position was stored for use by the neuronavigator in the remaining sessions. Biphasic TMS pulses were applied with a figure-of-eight coil (MC-B70) and a MagPro x 100 stimulator (MagVenture A/S). The individual threshold of cortical excitability was established as the lowest stimulation intensity applied over visual cortex necessary for inducing phosphenes after dark adaptation. The stimulation intensity used during the experiment was set at $120 \%$ of the individual dark-adapted phosphene threshold on early visual cortex, which ranged from 70 to $80 \%$ of the maximal stimulator output. Participants wore earplugs throughout the whole experiment.

\section{Results}

We found that none of these prevalent hypotheses could account for the data we report in its entirety, that is, for the respective shifts of the CRF induced by TMS with and without prior adaptation. Instead, we observed a decrease of contrast sensitivity with TMS in the no-adaptation state, but an increase of sensitivity with

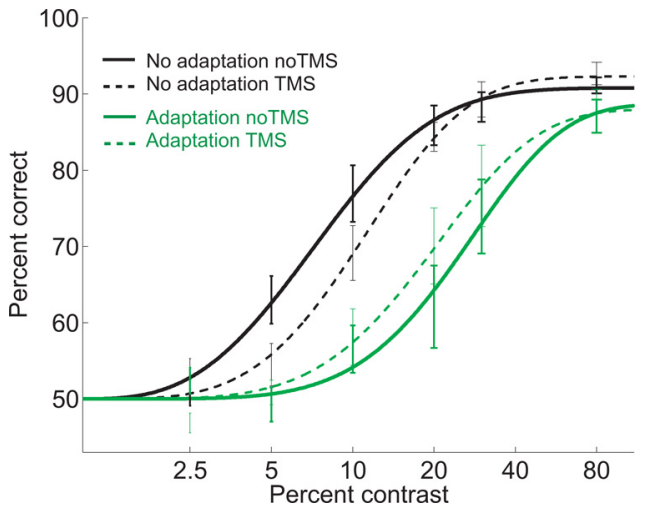

Figure 3. Adaptation and TMS effects. Accuracy was higher for the nonadapted (black lines) than the adapted (green lines) condition. TMS (dotted lines) had a different effect depending on the state of adaptation: it decreased accuracy for the nonadapted condition for intermediate contrast levels (dotted vs continuous black lines), but increased performance in the adapted condition (dotted vs continuous green lines). Bars are SEM.

TMS in the adaptation state for contrast levels in the dynamic range of the CRF.

The effects of adaptation, TMS, and contrast on the mean accuracy are depicted in Figure 3. We submitted accuracy to a three-way repeated-measures ANOVA with the application of TMS (TMS/no TMS), adaptation state (adaptation/no-adaptation), and contrast $(2.5,5,10,20,30$, and $80 \%)$ as within-subject factors. Accuracy was contrast dependent $\left(F_{(5,25)}=96.195, p<0.0001\right)$ with higher contrast levels leading to higher accuracy. Moreover, adaptation led to a strong reduction in performance, with an average accuracy of $75 \%$ for nonadapted and 64\% for adapted gratings $\left(F_{(1,5)}=32.038, p=\right.$ $0.002)$. This decrease due to adaptation was stronger for intermediate contrast levels (adaptation $\times$ contrast: $F_{(5,25)}=5.117, p=0.02$ ), confirming previous findings on contrast-adaptation (Dao et al., 2006; Pestilli et al., 2007). In addition, TMS had a different effect on accuracy depending on contrast level and adaptation (adaptation $X$ TMS $\times$ contrast level: $\left.F_{(5,25)}=6.108, p=0.001\right)$.

To differentiate among the three models (signal suppression and the two noise induction accounts), we obtained four psychometric CRFs for each observer (adaptation-no TMS, no adaptation-no TMS, adaptation-TMS, and no adaptation-TMS) by fitting Weibull functions (Weibull, 1951) to the data using the maximum likelihood procedure implemented in psignifit (Wichmann and Hill, 2001a,b), a freely available toolbox for MATLAB (Mathworks). For each observer, we estimated contrast thresholds at a fixed performance level of 70\% (halfway between chance and asymptote). We analyzed these fitted thresholds and asymptotic performance to assess the magnitude of the adaptation and TMS effect with or without prior adaptation, using a two-way repeated-measures ANOVA with TMS (TMS/no TMS) and adaptation state (adaptation/no-adaptation). TMS had a differential effect on thresholds with respect to adaptation state (TMS $\times$ adaptation, $\left.F_{(1,5)}=12.68, p=0.016\right)$. TMS significantly increased thresholds in nonadapted conditions $\left(t_{(5)}=-3.315\right.$, $p=0.02)$, but decreased thresholds after adaptation $\left(t_{(5)}=3.321\right.$, $p=0.02)$. These effects are also present in the individual thresholds (Fig. 4). TMS affected neither the asymptote nor the slope in any condition (TMS $\times$ adaptation, $F_{(1,5)}<1, p=0.62$; TMS $\times$ adaptation, $F_{(1,5)}<1, p=0.86$, respectively; Fig. 3).

\section{Discussion}

We assessed the effect of TMS on the behavioral contrast response function in six participants with and without prior con- 


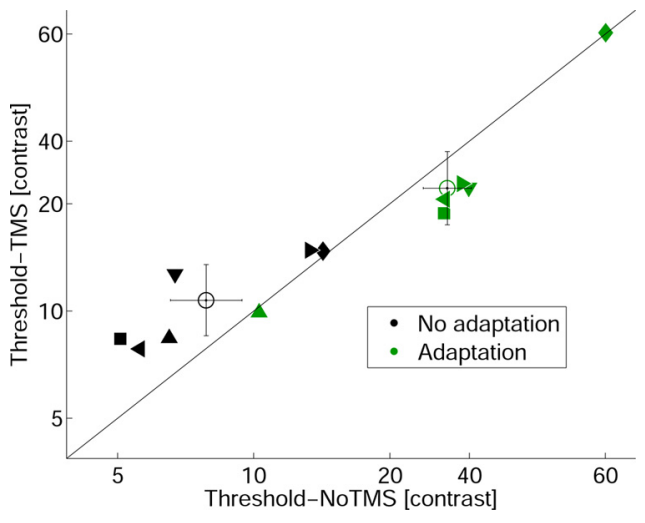

Figure 4. Scatter plots of thresholds for TMS and no-TMS conditions. Individual fitted threshold values are plotted for the no-TMS ( $x$-axes) versus the TMS ( $y$-axes) conditions. Black symbols represent nonadapted conditions, and green symbols represent adapted conditions. Each symbol depicts one observer. Open circles correspond to the averages of the six participants, with error bars representing the respective SEM. TMS systematically increased individual contrast thresholds in the nonadapted condition, while it decreased contrast thresholds in the adapted condition.

trast adaptation. Without TMS, we replicated the finding that contrast adaptation leads to a decrease of contrast sensitivity (Ohzawa et al., 1982; Bonds, 1991; Pestilli et al., 2007), yielding a rightward shift of the CRF (Fig. 3). With TMS, we found clear evidence for state dependency. Applying TMS decreased contrast sensitivity across the dynamic range when there was no adaptation, but TMS increased contrast sensitivity across the dynamic range when participants were contrast adapted. In addition, similarly to adaptation effects (Pestilli et al., 2007), TMS affected only the thresholds of psychometric functions, leaving asymptotes unchanged. According to the well established Naka-Rushton function (Naka and Rushton, 1966), which describes the CRF, such left-right shift of the curve without affecting the asymptotes can only be explained if the variable introduced in the system works at the input level of the function. In our study, this suggests that TMS acts at the input level of neuronal processing, i.e., at the synaptic level, via an input-gain mechanism. To understand how TMS differently affected behavioral sensitivity, we focus first on adaptation and then on TMS.

\section{Behavioral and neural effects of adaptation on contrast sensitivity}

The finding that adaptation shifts the CRF to the right without changing the asymptote has been taken to suggest that adaptation is a form of reduced contrast gain (Dao et al., 2006; Pestilli et al., 2007), which has also been reported with functional imaging in early visual cortex (Gardner et al., 2005). A similar CRF shift was also found in a physiological study of contrast adaptation using intracellular recordings in simple and complex cells of cat primary visual cortex (Carandini and Ferster, 1997). These authors found that spike rate increases with contrast and that after adaptation the resulting CRF was shifted to the right. Further investigations on membrane conductivity led them to conclude that the effect of adaptation is best explained by lack of excitation rather than an increase of tonic suppression, suggesting that adaptation changes synaptic efficacy.

\section{State-dependent effects of TMS Preadaptation}

In this study, we observed that in the nonadapted state TMS decreased contrast sensitivity. This finding is compatible with the pre- dominant view that TMS creates virtual lesions (hypothesis 1) by suppressing neuronal activity (Walsh and Rushworth, 1999) (Fig. $1 b)$ as well as with the noise induction account of TMS intensitydependent effects (Fig. 1e) (hypothesis 4). However, the TMSinduced decreased sensitivity in the nonadapted state is not consistent with the preferential activation of less active neurons view (Silvanto and Muggleton, 2008) (hypothesis 2) because this would predict a change in asymptote (Fig. 1c). Our data are also incompatible with the noise induction account of stimulus intensitydependent effects (hypothesis 3), for this would predict that noise would push up performance for subthreshold stimuli and pull down performance for suprathreshold stimuli, thus having a beneficial effect for the former but a detrimental effects for the latter (Fig. 1d). Note that the impairment in sensitivity brought about by TMS cannot be attributed to phosphene induction, because the effect would have been the same for the adapted and the nonadapted conditions.

Seemingly, our results are inconsistent with the conclusion reached by Ruzzoli et al. (2010). They conducted a study dealing with the effects of TMS in V5 on motion coherence and report that TMS leads to a decrement in sensitivity, depicted by a shallower slope. They interpret their results to be consistent with the hypothesis that TMS induces randomly distributed neural noise. We note, however, that their conclusion is based on average results, but that the results of only less than half of the participants are consistent with such a hypothesis.

\section{Postadaptation}

In the adapted state, TMS increased contrast sensitivity across the entire dynamic range of the CRF, shifting it leftward, because the effect was more pronounced at the intermediate levels. This finding is incompatible with all discussed hypotheses: (1) virtual lesion-it would always predict detrimental effects on behavior (Fig. 1b); (2) the preferential activation of less active neurons view (Silvanto and Muggleton, 2008) would predict a change in asymptote reflecting a stronger effect at higher contrast levels (Fig. 1c); (3) noise induction/stimulus intensity-it would predict beneficial effect for low stimulus contrast, but detrimental effects for high stimulus contrast (Fig. 1d); and (4) noise induction/TMS and stimulus intensity - it would predict a facilitatory effect for low to intermediate stimulus contrasts (Fig. 1e) with a consequent change in slope of the CRF. Schwarzkopf et al. (2011) have proposed that after adaptation TMS is less effective activating the neurons because they are less susceptible, and that "this is akin to reducing the TMS intensity" (Schwarzkopf et al., 2011, p. 3146). Thus, they reason, after adaptation the TMS would have a similar effect to that caused by reducing the TMS intensity, at which point stochastic resonance would be observed. According to this proposal, it would follow that without adaptation the TMS effect should be strong and therefore detrimental, but with adaptation the effect should be weak and therefore beneficial. Moreover, if TMS effects became increasingly smaller as stimulus signal increases, one would expect a stronger facilitation for lowto-intermediate contrast stimuli than for intermediate to highcontrast stimuli, leading to a shallower slope. We note two relevant differences between our study and the studies of Schwarzkopf et al. (2011) and Ruzzoli et al. (2010): (1) they did not manipulate the state of the neurons before stimulation, as in TMSa studies; and (2) they used three TMS pulses instead of one single pulse, with stimulus duration overlapping with one or two pulses. Future experiments should explicitly vary TMS intensity.

In sum, none of the proposed hypotheses can account simultaneously for the observed results with and without adaptation. Furthermore, phosphene induction cannot explain the present findings. 
It would decrease signal-to-noise ratio and consequently impair performance orientation discrimination in both adaptation conditions; however, accuracy increased after adaptation. Our results make it necessary to simultaneously explain the rightward shift without adaptation and the leftward shift with adaptation, suggesting that TMS may change the balance of excitation and inhibition. Moliadze et al. (2003) have reported that TMS pulses of high intensity (exceeding $50 \%$ of maximal stimulator output) suppress predominant activity during the first 100-200 ms. Based on our findings, we propose that TMS changes the balance of excitatory and inhibitory input, supporting the idea that TMS has a suppressive effect that is activity dependent, with more active neurons being more strongly affected. It has been shown that the predominant activation without adaptation is excitatory, but after adaptation excitation decreases (Carandini and Ferster, 1997). Thus, according to our hypothesis, in the nonadapted case the predominant excitatory input would be more suppressed by TMS than any inhibitory input, resulting in a rightward shift of the CRF and a decrease in performance. Conversely, in the adapted case, suppressing inhibitory activity by TMS would lead to disinhibition, yielding an increase of sensitivity and a leftward shift of the CRF.

Our hypothesis could also explain previous studies on statedependent TMS while disambiguating the hypothesis put forward by Silvanto et al. (2007) to account for the observation that after adaptation a TMS pulse leads to the perception of the adapter, rather than its opponent. In their view "TMS perceptually facilitates the attributes encoded by the less active neural population" (Silvanto et al., 2007), which would leave open the possibility that this perceptual facilitation is achieved either by exciting the less active neurons or by inhibiting the more active neurons. However, as we have argued above, unspecific excitation of less active neurons or unspecific inhibition of more active neurons both predict in our experiment a change in asymptote of the CRF, which we did not observe. We propose to treat excitatory and inhibitory neuronal populations separately, and that TMS has an activity-dependent suppressive effect on the inhibitory population. Consequently, we suggest that the increased contrast sensitivity brought about by TMS after adaptation results from an underlying mechanism of disinhibition rather than excitation.

\section{Conclusion}

The present study shows that TMS impairs perception when the visual cortex is not adapted, but facilitates perception after adaptation. These results cannot be accounted for by any of the current hypotheses underlying TMS effects. Here we propose that an activity-dependent suppressive effect of TMS may underlie state dependency of TMS effects, with TMS suppressing more the excitatory or the inhibitory neuronal population depending on the neuronal state at the time of stimulation.

\section{References}

Amassian VE, Cracco RQ, Maccabee PJ, Cracco JB, Rudell A, Eberle L (1989) Suppression of visual perception by magnetic coil stimulation of human occipital cortex. Electroencephalogr Clin Neurophysiol 74:458-462.

Bonds AB (1991) Temporal dynamics of contrast gain in single cells of the cat striate cortex. Vis Neurosci 6:239-255.

Brainard DH (1997) The psychophysics toolbox. Spat Vis 10:433-436.

Cameron EL, Tai JC, Carrasco M (2002) Covert attention affects the psychometric function of contrast sensitivity. Vision Res 42:949-967.

Carandini M, Ferster D (1997) A tonic hyperpolarization underlying contrast adaptation in cat visual cortex. Science 276:949-952.

Carrasco M, Penpeci-Talgar C, Eckstein M (2000) Spatial covert attention increases contrast sensitivity across the CSF: support for signal enhancement. Vision Res 40:1203-1215.
Cattaneo L, Sandrini M, Schwarzbach J (2010) State-dependent TMS reveals a hierarchical representation of observed acts in the temporal, parietal, and premotor cortices. Cereb Cortex 20:2252-2258.

Cattaneo Z, Silvanto J (2008) Time course of the state-dependent effect of transcranial magnetic stimulation in the TMS-adaptation paradigm. Neurosci Lett 443:82-85.

Dao DY, Lu ZL, Dosher BA (2006) Adaptation to sine-wave gratings selectively reduces the contrast gain of the adapted stimuli. J Vis 6:739-759.

Gardner JL, Sun P, Waggoner RA, Ueno K, Tanaka K, Cheng K (2005) Contrast adaptation and representation in human early visual cortex. Neuron 47:607-620.

Harris JA, Clifford CW, Miniussi C (2008) The functional effect of transcranial magnetic stimulation: signal suppression or neural noise generation? J Cogn Neurosci 20:734-740.

Herrmann K, Montaser-Kouhsari L, Carrasco M, Heeger DJ (2010) When size matters: attention affects performance by contrast or response gain. Nat Neurosci 13:1554-1559.

McAdams CJ, Maunsell JH (1999) Effects of attention on orientationtuning functions of single neurons in macaque cortical area V4. J Neurosci 19:431-441.

Moliadze V, Zhao Y, Eysel U, Funke K (2003) Effect of transcranial magnetic stimulation on single-unit activity in the cat primary visual cortex. J Physiol 553:665-679.

Nachmias J (1967) Effect of exposure duration on visual contrast sensitivity with square-wave gratings. J Opt Soc Am 57:421-427.

Naka KI, Rushton WA (1966) S-potentials from colour units in the retina of fish (Cyprinidae). J Physiol 185:536-555.

Ohzawa I, Sclar G, Freeman RD (1982) Contrast gain control in the cat visual cortex. Nature 298:266-268.

Pelli DG (1997) The VideoToolbox software for visual psychophysics: transforming numbers into movies. Spat Vis 10:437-442.

Pestilli F, Viera G, Carrasco M (2007) How do attention and adaptation affect contrast sensitivity? J Vis 7:9.1-9.12.

Pestilli F, Ling S, Carrasco M (2009) A population-coding model of attention's influence on contrast response: estimating neural effects from psychophysical data. Vision Res 49:1144-1153.

Ruzzoli M, Marzi CA, Miniussi C (2010) The neural mechanisms of the effects of transcranial magnetic stimulation on perception. J Neurophysiol 103:2982-2989.

Salminen-Vaparanta N, Noreika V, Revonsuo A, Koivisto M, Vanni S (2012) Is selective primary visual cortex stimulation achievable with TMS? Hum Brain Mapp 33:652-665.

Schwarzbach J (2011) A simple framework (ASF) for behavioral and neuroimaging experiments based on the psychophysics toolbox for MATLAB. Behav Res Methods 43:1194-1201.

Schwarzkopf DS, Silvanto J, Rees G (2011) Stochastic resonance effects reveal the neural mechanisms of transcranial magnetic stimulation. J Neurosci 31:3143-3147.

Silvanto J, Muggleton NG (2008) New light through old windows: moving beyond the "virtual lesion" approach to transcranial magnetic stimulation. Neuroimage 39:549-552.

Silvanto J, Muggleton NG, Cowey A, Walsh V (2007) Neural adaptation reveals state-dependent effects of transcranial magnetic stimulation. Eur J Neurosci 25:1874-1881.

Silvanto J, Muggleton N, Walsh V (2008) State-dependency in brain stimulation studies of perception and cognition. Trends Cogn Sci 12:447-454.

Simonotto E, Riani M, Seife C, Roberts M, Twitty J, Moss F (1997) Visual perception of stochastic resonance. Phys Rev Lett 78:1186-1189.

Stocks NG (2000) Suprathreshold stochastic resonance in multilevel threshold systems. Phys Rev Lett 84:2310-2313.

Thielscher A, Reichenbach A, Uğurbil K, Uludağ K (2010) The cortical site of visual suppression by transcranial magnetic stimulation. Cereb Cortex 20:328-338.

Walsh V, Rushworth M (1999) A primer of magnetic stimulation as a tool for neuropsychology. Neuropsychologia 37:125-135.

Weibull W (1951) A statistical distribution function of wide applicability. J Appl Mech 18:293-297.

Wichmann FA, Hill NJ (2001a) The psychometric function: I. Fitting, sampling, and goodness of fit. Percept Psychophys 63:1293-1313.

Wichmann FA, Hill NJ (2001b) The psychometric function: II. Bootstrap-based confidence intervals and sampling. Percept Psychophys 63:1314-1329. 